\title{
The role of combined spinal epidural analgesia for labour: is there still a question?
}

\author{
Roanne Preston MD FRCPC
}

W HEN combined spinal epidural (CSE) analgesia was introduced as a technique for labour analgesia, two distinct camps of opinion quickly emerged amongst anesthesiologists practicing obstetrical anesthesia: those who believe benefits of CSE are advantageous to every woman requesting a labour epidural; and those who believe the benefits over a standard epidural do not justify the intentional "invasion" of the dura nor the side effects. Efficacy has been well proven by a plethora of CSE studies, but safety remains a concern for many.

In this issue of the Journal, Rofaeel et al. ${ }^{1}$ report on a CSE study, examining hyperbaric bupivacaine $v s$ isobaric bupivacaine. The study finds that when added to intrathecal (IT) sufentanil $2.5 \mu \mathrm{g}$, isobaric bupivacaine $2.5 \mathrm{mg}$, the standard dose which many anesthesiologists use, provides better analgesia within the first ten minutes post injection, compared to a 2.5 - $\mathrm{mg}$ dose of hyperbaric bupivacaine. This difference disappears by 20 min post injection. The far more important finding of the study is that the incidence of sustained fetal bradycardia, defined as fetal heart rate $<100$ beats. $\mathrm{min}^{-1}$ for $>60 \mathrm{sec}$, was $33 \%$ in the isobaric group vs $10 \%$ in the hyperbaric group. This result is both statistically and clinically significant. While initial reports on CSE showed a high incidence of fetal bradycardia, more contemporary prospective studies, including those by Wong et al. ${ }^{2}$ in 2005 and Van de Velde et al. ${ }^{3}$ in 2004, report an incidence of prolonged decelerations varying between $3.9 \%-12 \%$. None of the fetal bradycardias reported in Rofaeel's study resulted in emergency obstetrical intervention, but perhaps this is a high cost to pay for a difference of ten minutes to attainment of excellent analgesia.

One might conclude from this study that IT isobaric bupivacaine should no longer be used. But, it raises a more important issue: are the benefits of CSE over a standard epidural for labour analgesia enough to justify this risk of fetal harm? As a provider of CSE labour analgesia, I have established my own set of exclusion criteria based on interpretation of the literature. For instance, like the authors of this study, I do not use the technique when there is a poor fetal heart trace, nor when I wish to know the epidural catheter is functioning unequivocally at the time of insertion. I also use CSE for those who may gain the most benefit: women in advanced labour and those in extreme pain. However, given the incidence of fetal bradycardia observed in both groups of Rofaeel's study, perhaps it is time to reassess whether CSE should continue to have a role in labour analgesia.

Today, the purported benefits of CSE are: faster onset of analgesia, superior analgesia in late labour, better subsequent functioning of the epidural catheter, and higher patient satisfaction. It has been well-proven that when compared to dilute epidural solutions, CSE offers no advantage in terms of obstetrical intervention, nor any difference in the ability to be mobile. ${ }^{4}$ The usually quoted disadvantages include: fetal bradycardia; unknown reliability of epidural catheter function; risk of central nervous system infection and respiratory depression; and significant pruritus. Risks of hypotension and post-dural puncture headache have been shown to be no different than with epidural analgesia. ${ }^{4}$

First, the question of benefits. Cochrane Systematic Reviews published in 2003 on CSE vs epidural, encompassing 14 trials with over 2,000 women, found that on average, onset of analgesia was 5.5 min faster with CSE. ${ }^{4}$ Other studies have shown similar results, many reporting a difference of 20-30 min to complete analgesia. ${ }^{5,6}$ While for the average woman requesting an epidural for labour a five- to ten-minute variance in onset time may not make much difference, for women approaching the second stage of labour, CSE provides

CAN J ANESTH $2007 / 54: 1 /$ pp 9-14

From the Department of Anesthesia, British Columbia Women's Hospital and Health Centre, and the Department of Anesthesiology, Pharmacology and Therapeutics, University of British Columbia, Vancouver, British Columbia, Canada.

Address correspondence to: Dr. Roanne Preston, Head, Department of Anesthesia, Rm 1Q72 BC Women's Hospital, 4500 Oak Street, Vancouver, B.C. V6H 3Nl, Canada. Phone: 604-875-2158; Fax: 604-875-2733; E-mail: rpreston@cw.bc.ca 
rapid sacral analgesia without sacrificing pushing power. ${ }^{7}$ How about the functioning of the epidural catheter? Several studies provide evidence that analgesia from the subsequent epidural infusion appears to be superior in women who received IT opioids. ${ }^{8,9}$ In addition, it appears that there may be fewer epidural catheter failures in women receiving CSE, perhaps because the appearance of CSF in the spinal needle confirms the position of the epidural needle. ${ }^{10}$ The other major benefit of CSE is increased patient satisfaction. The Cochrane Systematic Review found an odds ratio of 4.69 in favour of CSE over the standard epidural technique, reflecting what the majority of individual studies found. ${ }^{4}$

What is the scientific evidence of risk? When the technique of CSE was first introduced for labour analgesia, four cases of bacterial meningitis associated with CSE were reported within a short time frame. In one case the meningitis presented $14 \mathrm{hr}$ after an epidural blood patch, but the other cases occurred with uncomplicated CSE techniques. ${ }^{11}$ The problem is that the incidence of meningitis secondary to labour epidurals is unknown. Recently, there have been several published reports from large databases examining the incidence of severe neurological consequences of regional anesthesia, which provide reasonable comparisons for such rare events. Moen et al., ${ }^{12}$ in a Swedish study that encompassed 975,000 spinal blocks and 225,000 epidural blocks, reported 29 cases of meningitis. Twenty-three of those cases were associated with spinal blocks for an incidence of 1:43,000; six cases of meningitis were reported with epidural blocks for an incidence of 1:37,000. Of note, there were no cases of meningitis reported for obstetrical blocks. Loo et al. ${ }^{13}$ reported on severe neurological complications of regional techniques in obstetrics, finding five cases of meningitis associated with labour epidurals, and three with CSE for labour. ${ }^{13}$ Is the meningitis risk increased with CSE? Probably not, but one cannot be cavalier about intentional dural puncture; strict aseptic technique is more difficult to maintain in a labour room than in the operating room.

In the first few years of use, there were six case reports of respiratory arrest associated with IT sufentanil or fentanyl for labour analgesia. The doses of IT opioid medications were higher in all these cases than presently recommended and, in three cases, the patients had received $i v$ opioids within the preceding two to three hours. ${ }^{14,15}$

Whether CSE poses an increased risk of fetal bradycardia compared to a standard epidural has not been well-addressed in the literature, mainly because the majority of reported CSE studies involve relatively small patient cohorts. Uterine hypertonus appears to be a specific complication of IT opioid use, and more frequently, is the cause of the bradycardia rather than maternal hypotension. A meta-analysis published in 2002, involving over 2,000 women, found an odds ratio of 1.8 for occurrence of fetal bradycardia within the first $60 \mathrm{~min}$ of IT opioid administration vs neuraxial analgesia without IT opioids. ${ }^{16}$ The episodes of bradycardia were not, however, associated with an increase in the rate of emergency Cesarean deliveries. There may be a dose response of fetal heart rate (FHR) changes with IT sufentanil, as demonstrated by Van de Velde et al. ${ }^{3}$ In 296 labouring parturients, these investigators compared the response to IT sufentanil doses of $1.5 \mu \mathrm{g}$ and $7.5 \mu \mathrm{g}$, with a control group of patients receiving a low-dose epidural. The incidence of non-reassuring FHR was $24 \%$ in the high-dose group compared to $12 \%$ and $11 \%$ in the low IT and epidural groups, respectively. Recent literature supports the use of lower doses of IT sufentanil - 2 to $5 \mu \mathrm{g}$ - when combined with a small dose of bupivacaine. ${ }^{17}$ It is unknown whether any differences in risk exist between sufentanil and fentanyl when used as a part of the CSE technique.

There are other minor side effects, including pruritus, that are clearly more frequent with CSE than with epidural analgesia: an odds ratio of 2.79 was reported in the Cochrane Review ${ }^{4}$ and 1.7 in Mardirosoff et al.'s meta-analysis. ${ }^{16}$ This kind of side-effect should be part of the informed consent process; it is unlikely to change the mind of most women, but we should be prepared to treat the problem effectively should it arise.

From the current best evidence it appears that the advantages of CSE include: rapid onset of analgesia, high patient satisfaction, and reliable if not better subsequent epidural analgesia. The significant risk of the technique is early fetal bradycardia which, if nothing else, is an anxiety-provoking event; and the most common side effect is pruritus. Given this risk-benefit analysis, what would a reasonable woman choose if offered the choice of CSE $v s$ epidural analgesia? If she were in extreme pain, a five-minute faster onset time may seem worthwhile. If the patient had previously experienced a poor labour epidural, the lure of good early analgesia and possibly a better epidural might tip the scale. Should we offer CSE to all women, or be selective, and offer the technique only to women for whom there is clear benefit? Science appears to lean in the latter direction, demonstrated by the results of the meta-analysis which showed that only 28 women need to receive intrathecal opioids for one fetal bradycardia to occur. ${ }^{16}$ If anesthesiologists are to continue to offer CSE for labour analgesia, we must ensure that there is 
full awareness of the specific side-effects, while having protocols in place to deal with them, and providing reasonable disclosure with our patients regarding the risks.

Where should future research related to CSE be directed? Before embarking on further studies to determine which drug combinations for the technique of labour analgesia are best, we should focus on developing a better understanding of the mechanism of uterine hypertonus associated with IT opioids/local anesthetic combinations for CSE. We also need to determine if there are variances in the responses to different opioid $\mu$ agonists, specifically, sufentanil and fentanyl. From the outcomes of investigations in these areas as well as the existing body of literature, the most appropriate IT drug combinations can be rationalized and evaluated, for more detailed efficacy analysis and assessment of maternal and neonatal safety.

The quest for the "perfect labour epidural" is an ongoing one. Combined spinal-epidural analgesia is a useful technique to add to our armamentarium of labour analgesia options, as not all women can be treated with the same epidural recipe. Labour analgesia needs to be a balance between the woman's needs, safety for the mother/baby unit, and the resources and anesthesia/nursing expertise within a given unit. While we continue to refine the search for a better understanding of the role for CSE, women should be appropriately informed of the benefits and risks of the technique, and the necessary protocols should be in place to ensure the provision of safe and efficacious analgesia for the labouring parturient.

\section{Le rôle de l'analgésie rachidienne-péridurale combinée pour le tra- vail obstétrical : y a-t-il encore une question?}

Lors de l'introduction de l'analgésie rachidienne-péridurale combinée (RPC) comme technique d'analgésie pour le travail obstétrical, deux camps d'opinion distincts ont rapidement vu le jour parmi les anesthésiologistes pratiquant l'anesthésie obstétricale : ceux qui croient que les bienfaits d'une RPC sont avantageux pour toute femme ayant besoin d'une péridurale pour le travail, et ceux qui pensent que les bienfaits supplémentaires, par rapport à une péridurale standard, ne justifient ni «l'invasion» intentionnelle de la dure-mère ni les effets secondaires. L'efficacité a été prouvée à maintes reprises par une pléthore d'études au sujet de la RPC, mais pour beaucoup, la sécurité demeure une préoccupation.

Dans ce numéro du Journal, Rofaeel et al. ${ }^{1}$ font un compte-rendu d'une étude au sujet de la RPC qui compare la bupivacaïne hyperbare à la bupivacaïne isobare. L'étude a trouvé que, lorsqu'elle est ajoutée à du sufentanil intrathécal (IT) $2,5 \mu \mathrm{g}$, la bupivacaïne isobare $(2,5 \mathrm{mg}-$ la dose standard que de nombreux anesthésiologistes utilisent) offre une meilleure analgésie dans les dix premières minutes post-injection, qu'une dose de $2,5 \mathrm{mg}$ de bupivacaïne hyperbare. La différence disparaît vingt minutes après injection. Toutefois, la découverte majeure de l'étude est que l'incidence de bradycardie foetale soutenue, définie comme une fréquence cardiaque foetale $<100$ battements.min. ${ }^{-1}$ pour $>60 \mathrm{sec}$, est de $33 \%$ dans le groupe isobare vs $10 \%$ dans le groupe hyperbare. Ce résultat est important à la fois statistiquement et cliniquement. Alors que les premiers comptes-rendus de RPC démontraient une forte incidence de bradycardie foetale, des études prospectives plus récentes, y compris celles de Wong et al. ${ }^{2}$ en 2005 et de Van de Velde et $a l^{3}$ en 2004, rapportent une incidence de décélérations prolongées qui varient entre 3,9\% et $12 \%$. Aucune des bradycardies fotales dont il est question dans l'étude de Rofaeel n'a eu pour conséquence une intervention obstétricale d'urgence, mais le prix à payer est peut-être élevé pour une différence de dix minutes jusqu'à l'obtention d'une excellente analgésie.

Suite à cette étude, on pourrait conclure que la bupivacaïne isobare IT ne devrait plus être utilisée. Toutefois, une question plus importante est soulevée : les bienfaits d'une RPC par rapport à une péridurale standard pour une analgésie de travail obstétrical sontils suffisants pour justifier ce risque d'atteinte foetale ? Dans ma pratique, j'ai établi mon propre ensemble de critères d'exclusion, me basant sur l'interprétation de la littérature. Par exemple, tout comme les auteurs de cette étude, je n'utilise pas cette technique lorsque le tracé du cœur foetal est inquiétant, ni lorsque je désire savoir si le cathéter péridural fonctionne sans équivoque lors de l'insertion. J'utilise moi aussi la RPC pour les parturientes qui en gagnent le plus de bienfaits : les femmes au travail obstétrical avancé et celles en grande douleur. Cependant, étant donné l'incidence de bradycardie foetale observée dans les 
deux groupes de l'étude de Rofaeel, il est peut-être temps de réévaluer si la RPC doit continuer à tenir un rôle dans l'analgésie du travail obstétrical.

Actuellement, les prétendus bienfaits d'une RPC sont: début d'analgésie plus rapide, analgésie supérieure lors du travail obstétrical tardif, meilleur fonctionnement ultérieur du cathéter péridural et satisfaction accrue des patientes. Il a été bien prouvé que, lorsque comparée à des solutions péridurales diluées, la RPC n'offre aucun avantage en termes d'intervention obstétricale, et qu'il n'existe aucune différence dans la capacité à être mobile. ${ }^{4}$ Les désavantages généralement cités comprennent: la bradycardie foetale, la fiabilité inconnue de la fonction du cathéter péridural, le risque d'une infection du système nerveux central, la dépression respiratoire, et un prurit important. Les risques d'hypotension et de céphalée post-ponction durale ont été démontrés équivalents à ceux d'une analgésie péridurale. ${ }^{4}$

En premier lieu, la question des bénéfices. Cochrane a publié un article de synthèse en 2003 comparant la RPC à la péridurale, basé sur 14 études incluant un total de plus de 2000 femmes; on a trouvé qu'en moyenne, le début de l'analgésie était plus rapide de 5,5 min avec la RPC. ${ }^{4}$ D'autres études ont montré des résultats similaires, dont nombre rapportaient une différence de 20-30 min. jusqu'à l'analgésie totale. ${ }^{5,6}$ Bien que, pour une femme moyenne demandant une péridurale pour le travail obstétrical, une variation de cinq à dix minutes jusqu'au début de l'analgésie ne fasse pas une grande différence, pour les femmes s'approchant de la deuxième phase de travail, la RPC fournit une analgésie sacrale rapide sans pour autant sacrifier la force d'expulsion. ${ }^{7}$ Qu'en est-il du fonctionnement du cathéter péridural ? De nombreuses études apportent des preuves que l'analgésie par perfusion péridurale ultérieure semble être supérieure chez les femmes ayant reçu des opiacés IT. ${ }^{8,9}$ En outre, il semble y avoir moins d'échecs de cathéter péridural chez les femmes recevant une RPC, peut-être parce que l'apparition de LCR dans l'aiguille rachidienne confirme la position de l'aiguille péridurale. ${ }^{10}$ L'autre avantage majeur de la RPC est la satisfaction accrue des patientes. L'article de synthèse Cochrane a découvert un rapport de cotes de 4,69 en faveur de la RPC par rapport à la technique péridurale standard, reflétant ce que la majorité des études individuelles a découvert. ${ }^{4}$

Y a-t-il preuve scientifique d'un risque ? Lors de la première introduction de la technique de RPC pour l'analgésie pendant le travail obstétrical, quatre cas de méningite bactérienne associée à la RPC ont été signalés dans un court laps de temps. Dans un des cas, la méningite s'est présentée $14 \mathrm{~h}$ après une péridurale avec sang homologue pour traiter une céphalée post ponction de la dure-mère, mais les autres cas se sont présentés avec des techniques de RPC sans complications. ${ }^{11}$ Le problème est que l'incidence de méningite secondaire aux péridurales pour travail obstétrical est inconnue. Plusieurs comptes-rendus issus d'importantes bases de données examinant l'incidence de complications neurologiques sévères imputables à une anesthésie régionale ont été publiés récemment; ces comptes-rendus fournissent des comparaisons raisonnables de ces rares événements. Moen et al., ${ }^{12}$ dans une étude suédoise englobant 975 000 blocs rachidiens et 225000 blocs périduraux, ont trouvé 29 cas de méningite. Vingt-trois de ces cas étaient associés à des blocs rachidiens, pour une incidence de $1: 43000$; six cas de méningite ont été déclarés avec des blocs périduraux, pour une incidence de $1: 37000$. Il est à noter qu'aucun cas de méningite n'était associé à des blocs obstétricaux. L'étude de Loo et $a l .{ }^{13}$ fait état de complications neurologiques sévères lors de techniques régionales en obstétrique et met en évidence cinq cas de méningite associés à des péridurales pour travail obstétrical, et trois avec des RPC pendant le travail. ${ }^{13}$ Le risque de méningite est-il accru lors de RPC ? Probablement pas, mais on ne peut être désinvolte lorsqu'il s'agit de ponction intentionnelle de la dure-mère ; il est plus difficile de maintenir une technique aseptique dans une salle de travail obstétrical que dans un bloc opératoire.

Durant les premières années d'utilisation, six cas d'arrêt respiratoire associés au sufentanil ou au fentanyl IT pour l'analgésie du travail obstétrical sont relatés. Dans tous ces cas, les doses d'opiacés IT étaient supérieures aux doses actuellement recommandées et, dans trois cas, les patientes avaient reçu des opiacés intraveineux dans les deux ou trois heures précédentes. ${ }^{14,15}$

La question de savoir si la RPC comprend davantage de risque de bradycardie foetale qu'une péridurale standard a été mal adressée dans la littérature, et ce principalement parce que la majorité des études portant sur la RPC relatées ne concernent que des groupes relativement petits de patientes. L'hypertonie utérine semble être une complication spécifique à l'utilisation des opiacés IT et est plus fréquemment la cause de bradycardie que l'hypotension maternelle. Une méta-analyse publiée en 2002 , portant sur plus de 2000 femmes, a découvert un rapport de cotes de 1,8 concernant l'incidence de bradycardie foetale durant les 60 premières minutes suivant l'administration d'un opiacé IT $v s$ une analgésie neuraxiale sans opiacés IT. ${ }^{16}$ Toutefois, les épisodes de bradycardie ne sont pas 
associés à une augmentation du taux d'accouchement en urgence par césarienne. Il pourrait y avoir un effet de dose réponse au niveau du changement de fréquence cardiaque foetale (FCF) avec le sufentanil IT, comme le démontrent Van de Velde et al. ${ }^{3}$ Ces chercheurs ont comparé la réaction à des doses de sufentanil IT de 1,5 $\mu \mathrm{g}$ et 7,5 $\mu \mathrm{g}$ de 296 parturientes en travail, avec un groupe témoin de patientes recevant une péridurale de faible dose. L'incidence de FCF inquiétante était de $24 \%$ dans le groupe à forte dose comparée à $12 \%$ et $11 \%$ dans les groupes à faible dose IT et témoin, respectivement. La littérature récente encourage l'utilisation de doses plus faibles de sufentanil IT - 2 à $5 \mu \mathrm{g}$ - lorsque celui-ci est combiné à une faible dose de bupivacaïne. ${ }^{17}$ Les différences de risque entre l'utilisation de sufentanil ou de fentanyl dans une technique de RPC sont inconnues.

Il existe d'autres effets secondaires mineurs, y compris le prurit, qui sont clairement plus fréquents avec une RPC qu'avec une analgésie péridurale : il est fait état d'un rapport de cotes de 2,79 dans l'article de synthèse Cochrane $e^{4}$ et de 1,7 dans la méta-analyse de Mardirosoff et al. ${ }^{16}$ Ce type d'effet secondaire devrait faire partie du processus de consentement éclairé ; il est improbable que cela fasse changer d'avis la plupart des femmes, mais nous devrions être prêts, le cas échéant, à traiter le problème de façon efficace.

Si nous considérons les meilleures données probantes disponibles actuellement, il semble que les avantages de la RPC soient : début rapide de l'analgésie, grande satisfaction des patientes, et analgésie péridurale ultérieure plus fiable, sinon meilleure. Le risque significatif de la technique est une bradycardie foetale précoce qui, même mineure, demeure un événement angoissant; le prurit est l'effet secondaire le plus courant. Au vu de cette analyse risque/bénéfice, que déciderait une femme raisonnable si elle devait choisir entre une RPC ou une analgésie péridurale? Si elle souffre beaucoup, un début d'analgésie plus rapide de cinq minutes pourrait sembler avantageux. Si la patiente a au préalable eu une mauvaise expérience de péridurale pour le travail obstétrical, l'attrait d'une bonne analgésie précoce et d'une péridurale potentiellement meilleure pourrait faire pencher la balance. Devrions-nous proposer une RPC à toutes les femmes, ou être sélectifs et l'offrir seulement aux femmes pour lesquelles les bénéfices sont sans équivoque ? La science semble privilégier la seconde alternative, étant donné les résultats de la méta-analyse qui montraient que seulement 28 femmes ont eu besoin de recevoir des opiacés intrathécaux pour que survienne un épisode de bradycardie foetale. Si nous souhaitons que les anesthésiologistes continuent à offrir une RPC pour l'analgésie du travail obstétrical, nous devons nous assurer que les effets secondaires spécifiques sont clairement compris, et ce tout en mettant en place des protocoles pour les gérer et en fournissant une information raisonnable à nos patientes concernant les risques.

Quelle est la direction à adopter pour la recherche future concernant la RPC ? Avant de se lancer dans des études plus approfondies visant à déterminer quelles combinaisons médicamenteuses sont les mieux adaptées à la technique d'analgésie du travail obstétrical, nous devrions nous concentrer sur le développement d'une meilleure compréhension du mécanisme d'hypertonie utérine associée aux combinaisons d'opiacés IT / d'anesthésie locale pour la RPC. Il faut également déterminer s'il existe des variations dans les réactions à différents agonistes opiacés $\mu$, notamment le sufentanil et le fentanyl. En se basant sur les résultats d'études dans ces domaines et sur la littérature existante, les combinaisons de médicaments IT les plus appropriées peuvent être rationalisées et évaluées, pour une analyse plus détaillée de l'efficacité et une évaluation de la sécurité maternelle et néonatale.

La quête de la «péridurale de travail obstétrical parfaite » est sans relâche. L'analgésie rachidiennepéridurale combinée est une technique utile à ajouter à notre arsenal thérapeutique pour l'analgésie du travail obstétrical, étant donné que toutes les femmes ne peuvent pas être traitées avec la même recette péridurale. L'analgésie du travail obstétrical se doit d'être un équilibre entre les besoins de la femme, la sécurité pour l'unité mère/enfant, et les ressources ainsi que l'expertise en anesthésie et soins infirmiers d'une unité donnée. Pendant que nous continuons à perfectionner la recherche d'une compréhension plus approfondie du rôle de la RPC, les femmes devraient être correctement informées des risques et bénéfices de la technique, et les protocoles nécessaires devraient être en place afin d'assurer une analgésie sûre et efficace pour la parturiente en travail.

\section{References}

1 Rofaeel A, Lilker S, Fallah S, Goldszmidt E, Carvalho J. Intrathecal plain vs hyperbaric bupivacaine for labour analgesia: efficacy and side effects. Can J Anesth 2007; 54: 15-20.

2 Wong CA, Scavone BM, Peaceman AM, et al. The risk of cesarean delivery with neuraxial analgesia given early versus late in labor. N Engl J Med 2005; 352: 655-65.

3 Van de Velde M, Teunkens A, Hanssens M, Vandermeersch E, Verhaeghe J. Intrathecal sufentanil and fetal heart rate abnormalities: a double-blind, double placebo-controlled trial comparing two forms 
of combined spinal epidural analgesia with epidural analgesia in labor. Anesth Analg 2004; 98: 1153-9.

4 Hughes D, Simmons SW, Brown J, Cyna AM. Combined spinal-epidural versus epidural analgesia in labour. Cochrane Database Syst Rev 2003: CD003401.

5 Collis RE, Davies DW, Aveling W. Randomised comparison of combined spinal-epidural and standard epidural analgesia in labour. Lancet 1995; 345: 1413-6.

6 Wilson MJ, Cooper G, MacArthur C, Shennan A; Comparative Obstetric Mobile Epidural Trial (COMET) Study Group UK. Randomized controlled trial comparing traditional with two "mobile" epidural techniques: anesthetic and analgesic efficacy. Anesthesiology 2002; 97: 1567-75.

7 Abouleish A, Abouleish E, Camann W. Combined spinal-epidural analgesia in advanced labour. Can J Anaesth 1994; 41: 575-8.

8 Sia AT, Camann WR, Ocampo CE, Goy RW, Tan HM, Rajammal S. Neuraxial block for labour analgesia-is the combined spinal epidural (CSE) modality a good alternative to conventional epidural analgesia? Singapore Med J 2003; 44: 464-70.

9 Leighton BL, Arkoosh VA, Huffnagle S, Huffnagle HJ, Kinsella SM, Norris MC. The dermatomal spread of epidural bupivacaine with and without prior intrathecal sufentanil. Anesth Analg 1996; 83: 526-9.

10 Norris $M C$. Are combined spinal-epidural catheters reliable? Int J Obstet Anesth 2000; 9: 3-6.

11 Harding $S A$, Collis RE, Morgan BM. Meningitis after combined spinal-extradural anaesthesia in obstetrics. $\mathrm{Br}$ J Anaesth 1994; 73: 545-7.

12 Moen V, Dablgren N, Irestedt L. Severe neurological complications after central neuraxial blockades in Sweden 1990-1999. Anesthesiology 2004; 101: 950-9.

13 Loo CC, Dablgren G, Irestedt L. Neurological complications in obstetric regional anaesthesia. Int J Obstet Anesth 2000; 9: 99-124.

14 Lu JK, Manullang TR, Staples MH, Kem SE, Balley $P L$. Maternal respiratory arrests, severe hypotension, and fetal distress after administration of intrathecal, sufentanil, and bupivacaine after intravenous fentanyl. Anesthesiology 1997; 87: 170-2.

15 Ferouz F, Norris MC, Leighton BL. Risk of respiratory arrest after intrathecal sufentanil. Anesth Analg 1997; 85: 1088-90.

16 Mardirosoff C, Dumont L, Boulvain M, Tramer MR. Fetal bradycardia due to intrathecal opioids for labour analgesia: a systematic review. BJOG 2002; 109: 27481 .

17 Wong CA, Scavone BM, Loffredi M, Wang WY, Peaceman AM, Ganchiff JN. The dose-response of intrathecal sufentanil added to bupivacaine for labor analgesia. Anesthesiology 2000; 92: 1553-8.

CAN J ANESTH 54: 1 www.cja-jca.org January, 2007 